\title{
La transformación digital de los centros escolares. Obstáculos y resistencias
}

\author{
Manuel Area Moreira \\ manarea@ull.edu.es \\ Pablo Joel Santana Bonilla \\ psantana@ull.edu.es \\ Ana L. Sanabria Mesa \\ asanabri@ull.edu.es \\ EDULLAB, Universidad de La Laguna, España
}

\begin{abstract}
Resumen
En este artículo se presenta el diseño y resultados de un estudio de casos sobre 40 centros públicos de Educación Infantil y Primaria, y de Educación Secundaria, en el que se analizó el nivel o estadio de integración pedagógica y organizativa de las tecnologías digitales en cada uno de los mismos. Para el análisis de los datos se elaboró una matriz de valoración con cuatro estadios o fases de transformación digital (iniciación, aplicación, integración, transformación) y ocho dimensiones de integración pedagógica y organizativa de las TIC. Los principales factores inhibidores u obstáculos identificados fueron: la escasez de dispositivos suficientes para el alumnado y el profesorado; conectividad limitada; la inestabilidad de claustro; la falta de liderazgo que impulsara el uso de las TIC en el centro; y la ausencia de una visión compartida del equipo directivo acerca del potencial educativo de las TIC.
\end{abstract}

\section{Palabras clave}

TIC, transformación digital, tecnología educativa, centro educativo, estudio de caso 


\title{
The digital transformation of schools. Obstacles and resistances
}

\author{
Manuel Area Moreira \\ manarea@ull.edu.es \\ Pablo Joel Santana Bonilla \\ psantana@ull.edu.es \\ Ana L. Sanabria Mesa \\ asanabri@ull.edu.es \\ EDULLAB, Universidad de La Laguna, España
}

\begin{abstract}
This article presents the design and results of a case study on 40 primary and secondary state schools undertaken to analyze the level or stage of pedagogical and organizational integration of digital technologies in each one of them. An evaluation matrix was developed for data analysis, made up by four stages or phases of digital transformation (initiation, application, integration, transformation) and eight dimensions of pedagogical and organizational integration of ICT. The main inhibiting factors or obstacles identified were: the shortage of devices for students and teachers; limited connectivity; the instability of the faculty; the lack of leadership that will drive the use of ICT in the school; and the lack of a shared vision of the school management team about the educational potential of ICT.
\end{abstract}

\section{Keywords}

ICT, digital transformation, educational technology, school, case study 


\section{Introdución}

La transformación digital de cualquier organización es un proceso complejo y, en ocasiones, disruptivo que inevitablemente pasa por distintas fases o estadios (Gale \& Aarons, 2018). En dicho proceso se entrecruzan factores de distinta naturaleza (tecnológicos, organizativos y culturales) diferenciales en cada institución (Matt, Hess \& Benlian, 2015; Leignel, Ungaro \& Staar, 2016). En este artículo pretendemos responder a la pregunta ¿en qué consiste y cuáles son los factores que inciden en que el proceso de transformación digital sea más lento o poco exitoso en unos centros escolares respecto a otros? ¿Cuáles son los obstáculos y resistencias comunes en aquellos centros educativos con un bajo nivel de transformación digital y pedagógica?

Existe, desde hace años, una relevante producción bibliográfica de estudios que ha explorado los procesos de incorporación e integración organizativa y pedagógica de las TIC en las aulas y los centros escolares. Sobre este particular, desde hace más de una década, disponemos de conocimiento acumulado consecuencia de variadas investigaciones donde se han explorado los factores y variables que condicionan positiva o negativamente los procesos de cambio e innovación educativa con las tecnologías en el contexto escolar.

Una de las fuentes de referencia pioneras fue el estudio internacional Second Information Technology in Education Study (SITES) llevado a cabo en 26 países dirigido por Pelgrum (2001) en el que se exploraron distintas variables con relación a la utilización de las tecnologías digitales en los centros escolares tanto de Educación Primaria como Secundaria, y en particular, sobre los obstáculos que limitan el potencial innovador o integración pedagógica de las TIC obtenidos a través de encuestas a docentes.

Otro estudio empírico relevante fue el informe sobre la utilización de las TIC en las escuelas europeas (Balanskat, Blamire y Kefala, 2006) en el que se identificaron como factores negativos: la ausencia de habilidades TIC o competencia digital, la baja motivación, la falta de confianza y la formación inadecuada del profesorado. Asimismo, se encontró que dichas barreras, a nivel de centro, se vincularon con la falta de infraestructura de TIC (baja calidad y mantenimiento inadecuado del hardware, acceso limitado a equipos de TIC, falta de capacitación en uso del software) y la ausencia de experiencias en desarrollo de proyectos educativos apoyados en el uso de las TIC. Un tercer tipo de obstáculos, según dicho informe, tiene que ver con la estructura rígida de los sistemas educativos tradicionales (currículum disciplinar, estructuras organizativas rígidas, así como metodologías de enseñanza y las evaluaciones tradicionales).

De forma paralela también se han elaborado distintos trabajos de revisión de la literatura publicada sobre esta problemática. Uno de los primeros fue el de la British Educational Communications and Technology Agency (2004) en el que se evidenció que los factores que inciden en la no utilización educativa de las TIC corresponden a la falta de confianza y ansiedad del profesorado ante las mismas, la ausencia de competencia y formación del docente, problemas de acceso a los recursos (ausencia de hardware y software, mala organización de los recursos, artefactos inadecuados), carencia de tiempo, los problemas técnicos, y las resistencias al cambio junto con actitudes negativas ante la tecnología.

Hew y Brush (2007) realizaron un meta-análisis de 48 estudios donde examinaron las barreras hacia la integración de la tecnología en la enseñanza-aprendizaje a nivel K-12. Estos autores hallaron que la integración de las TIC está directamente afectada por las actitudes y creencias de los docentes, así como la falta de conocimiento y competencias tecnológicas, la carencia de dominio para el uso pedagógico de la tecnología y para la gestión del aula, la falta de habilidades de liderazgo, la ausencia de un plan de integración tecnológica adecuadamente temporalizado para permitir al centro asumir y organizar una visión global y compartida, así como la falta de recursos tecnológicos o de acceso a los mismos y de servicios de apoyo técnico. 
Keengwe, Onchwari y Wachira (2008), por su parte, señalan que

las principales barreras identificadas por los docentes en el uso e integración de la tecnología informática en el aula incluyen: falta de computadoras y software de calidad relevante, falta de tiempo, falta de financiación, problemas técnicos, actitud docente hacia las computadoras, falta de confianza del profesorado, resistencia al cambio, poco apoyo administrativo, poca capacitación y falta de visión para integrar la tecnología en el plan de estudios. (pg. 562)

Más recientemente Kopcha (2012) revisando dichas barreras las sintetizó en falta de acceso, visión estratégica, creencias pedagógicas, tiempo disponible y desarrollo profesional inadecuado.

En definitiva, existe un amplio conjunto de trabajos empíricos que ha examinado e identificado los principales obstáculos que provocan la ausencia o fracaso de la innovación educativa con las tecnologías y que, como sintetizó Ertmer (1999) se corresponde a dos grandes tipos de factores: a) factores externos y fuera de control docente como son el acceso a la tecnología, la disponibilidad de tiempo y soporte técnico, recursos, contenido y capacitación; y b) barreras o factores internos bien a nivel escolar, como la cultura organizacional, bien a nivel docente, como son sus creencias sobre la enseñanza con la tecnología así como su grado o capacidad de apertura al cambio e innovación.

Casi todos los trabajos citados se han centrado en la exploración del uso de las TIC a nivel del aula y desde la perspectiva del profesorado. Sin embargo, nos falta obtener más evidencias de cómo un centro escolar, en cuanto institución educativa compleja, es capaz de innovarse pedagógicamente y transformarse digitalmente (Law, Yuen y Fox, 2011; Valverde, 2015). Sobre esta problemática el número de publicaciones académicas es más limitado. Los trabajos que han abordado esta línea de investigación han pretendido identificar distintas fases o etapas evolutivas del centro como organización que progresa desde un estadio inicial de llegada de las TIC con poca innovación pedagógica hasta otros más evolucionados donde el centro se transforma digitalmente (Severin, 2011; Lugo y Kelly, 2011; Severin, Peirano y Falck, 2012). En este sentido es destacable el trabajo del Joint Research Center (JRC) con vistas a establecer un marco europeo de organizaciones digitalmente competentes (Kampylis, Punie \& Devine, 2015).

En esta dirección o línea de investigación hemos desarrollado un estudio empírico en el contexto del sistema escolar de las Islas Canarias elaborado con la finalidad de identificar los factores y variables que inciden en el grado, estadio o fase evolutiva de un centro escolar con relación a su transformación digital prestando una especial atención a aquellos factores que explican o están asociados con la ausencia o débil integración pedagógica y organizativa de las TIC.

\section{Un estudio sobre el grado de integración organizativa y pedagógica de las TIC en los centros escolares}

\section{a. Contexto del estudio}

Este estudio fue desarrollado en el marco de la evaluación del programa TSP (Tecnologías al Servicio de las Personas) destinado a la dotación y uso de las tecnologías digitales en el sistema escolar en Canarias. La evaluación de dicho programa se realizó como un contrato de investigación entre la Fundación General de la Universidad de La Laguna y Consejería de Educación, Universidades y Sostenibilidad (CEUS) del Gobierno de Canarias., obtenido en concurso público. El equipo técnico responsable de la investigación fue el grupo EDULLAB (Laboratorio de Educación y Nuevas Tecnologías) de la Universidad de La Laguna. Una síntesis del conjunto de estudios realizados de la evaluación del programa TSP puede consultarse en Area (2015). Se desarrollaron 
cinco estudios en los que se exploraron las visiones de los agentes educativos (profesorado, alumnado, agentes técnicos de apoyo, coordinadores TIC de centro) y las prácticas de uso de las TIC en los centros escolares.

El diseño y resultados presentados en este artículo se corresponden con el estudio cuarto de la citada evaluación y fue desarrollado con un enfoque metodológico de aproximación cualitativa (estudio de casos) en el que se exploró la situación o estadio de la transformación digital en cuarenta centros públicos de ambas provincias canarias y de distintos niveles educativos (Infantil y Primaria, y Secundaria) del sistema escolar de esta comunidad autónoma.

\section{b. Los objetivos del estudio}

a) Realizar un diagnóstico sobre el nivel de integración pedagógica y organizativa de las TIC en centros escolares públicos explorando:

- la disponibilidad de recursos e infraestructuras tecnológicas,

- el uso pedagógico de las TIC en los distintos espacios de los centros educativos (tareas, frecuencias uso, fines, prácticas o actividades realizadas),

- la valoración y expectativas de los agentes educativos del centro (equipo directivo, coordinador/a TIC, docentes, AMPA) hacia las TIC en educación, y

- los formatos de comunicación y gestión de la información en la comunidad educativa del centro mediante las TIC.

b) Identificar aquellos factores o fenómenos que afectan de forma positiva o negativa a los procesos de transformación digital y educativa comunes a varios centros y que permiten comprender los fenómenos que impulsan o inhiben dichos procesos.

Un tercer objetivo, del que no se da cuenta en este artículo, fue realizar recomendaciones y propuestas de actuación a corto y medio plazo para la política educativa destinada a facilitar la mejora de la integración escolar de las tecnologías en los centros educativos del Archipiélago.

\section{c. La muestra}

La muestra estuvo compuesta por 40 centros distribuidos según aparece en la Tabla 1. La selección de la muestra fue consensuada entre el equipo investigador y los responsables del Proyecto Tecnología al Servicio de las Personas (TSP) de la Consejería de Educación, Universidades y Sostenibilidad (CEUS) del Gobierno de Canarias. Los criterios de selección fueron los siguientes: que los centros estuvieran desarrollando algún proyecto de integración de las TIC, abarcar las diversas etapas educativas (Educación Infantil, Primaria, Secundaria y Bachillerato) y que hubiera centros de todas las islas. La Tabla 1 recoge la distribución por tipo de centro. La distribución por islas fue la siguiente: 3 centros de la isla de El Hierro, 3 de Fuerteventura, 12 de Gran Canaria, 3 de La Gomera, 3 de Lanzarote, 3 de La Palma y 13 de Tenerife.

\begin{tabular}{|l|l|l|l|}
\hline CEIP & CEO & IES & Total \\
Centros de \\
$\begin{array}{l}\text { Educación } \\
\text { Infantil y } \\
\text { Primaria }\end{array}$ & $\begin{array}{l}\text { Enseñanza } \\
\text { Obligatoria }\end{array}$ & $\begin{array}{l}\text { Institutos de } \\
\text { Educación } \\
\text { Secundaria }\end{array}$ & \\
\hline 21 & 2 & 17 & 40 \\
\hline
\end{tabular}

Tabla 1. Muestra por tipo de centro 


\section{d. Los instrumentos de recogida de información}

A continuación, se enumeran las técnicas e instrumentos diseñados específicamente para la recogida de información.

- Ficha de datos de identificación del centro, con indicadores sobre el tipo del centro y su localización; el número de unidades, de alumnado y de personal docente y no docente; la situación laboral de los docentes, su experiencia profesional y la antigüedad en el centro; y por último la disponibilidad o no de coordinador/a TIC.

- Ficha de recursos digitales generados por el centro: web, blog, redes sociales del centro, departamentos, o asignaturas.

- Entrevistas semiestructuradas al equipo directivo del centro, coordinadores TIC y profesorado que participó en la observación de aula.

- La entrevista al equipo directivo de los centros estaba organizada en torno a las siguientes dimensiones: formación y experiencia del equipo, implicación del profesorado en relación a las TIC e integración de las TIC en el centro

- La entrevista al coordinador/a TIC estaba estructurada en las siguientes dimensiones: antecedentes del uso de las TIC en el centro, el uso actual de las mismas, papel del coordinador TIC, el Plan TIC y la formación del profesorado.

- Observaciones de aula con un guion semiestructurado en torno a los siguientes indicadores: la actividad del alumnado, el rol del docente, los recursos utilizados (hardware/software y on line/off line) y quien los utiliza, y por último la organización del aula.

- Cuestionario a la Junta Directiva de la Asociación de Padres y Madres de Alumnado (AMPA) del centro: opinión y valoración sobre el uso de las TIC en el centro y aulas y sobre el equipamiento con el que cuenta el centro; información, participación e implicación en las actividades sobre las TIC en el centro; actividades de formación TIC desarrolladas.

- Guion análisis del Plan TIC, atendiendo a los indicadores: trayectoria innovadora del centro, dotación y organización de los recursos tecnológicos, estructura organizativa para la dinamización del plan, los objetivos, estrategias y acciones del plan, y evaluación de las acciones desarrolladas.

- Diario del investigador, atendiendo a los indicadores identificativos del centro, los descriptivos de las sesiones, y a aspectos valorativos referidos al desarrollo del proceso de recogida de información.

\section{e. El proceso de análisis de la información}

El proceso de análisis de la información se realizó en dos fases: primero se analizaron los datos centro por centro elaborándose un informe específico para cada caso (en total, cuarenta informes), identificando (a) los niveles de integración de las TIC de cada centro y (b) los factores que facilitaban o dificultaban tal integración en cada caso; en segundo lugar, se realizó un análisis comparativo entre los centros. Los resultados de este artículo corresponden al producto del análisis comparativo entre casos.

Para el análisis de la información y la elaboración del informe de cada centro se siguió un índice común (Tabla 2).

\begin{tabular}{|l|l|}
\hline Dimensiones & Sub-dimensiones \\
\hline \multirow{3}{*}{ Características generales del centro } & $\begin{array}{l}\text { - Características descriptivas del centro } \\
\end{array}$ \\
& - Trayectoria innovadora del centro \\
& - Trayectoria con respecto a las TIC \\
\hline Organización y gestión de los & - Disponibilidad, adquisición y mantenimiento \\
\hline
\end{tabular}




\begin{tabular}{|c|c|}
\hline recursos tecnológicos del centro & - Organización y distribución \\
\hline $\begin{array}{l}\text { Política interna del centro respecto a las } \\
\text { TIC }\end{array}$ & $\begin{array}{l}\text { - Características, proceso de elaboración y } \\
\text { grado de desarrollo del Plan TIC } \\
\text { - Liderazgo del Equipo Directivo en } \\
\text { relación al impulso de las TIC } \\
\text { - Coordinación, apoyo y asesoramiento } \\
\text { a la integración pedagógica de las TIC } \\
\text { - Competencia y formación del profesorado } \\
\text { para el uso pedagógico de las TIC } \\
\text { - La visibilidad del centro en Internet } \\
\text { - La comunicación y participación del AMPA } \\
\text { en la política TIC del centro }\end{array}$ \\
\hline Prácticas del centro con TIC & $\begin{array}{l}\text { - Proyectos propios que desarrolla el centro } \\
\text { con TIC } \\
\text { - Participación del centro en otros } \\
\text { proyectos, experiencias o redes } \\
\text { educativas on line } \\
\text { - Tipo de uso de las TIC en las aulas } \\
\text { - Producción y gestión de recursos digitales } \\
\text { para la enseñanza y aprendizaje } \\
\text { - Uso de las TIC para tareas administrativas, } \\
\text { de gestión y de comunicación entre el } \\
\text { profesorado del centro }\end{array}$ \\
\hline $\begin{array}{l}\text { Visiones de los agentes educativos: equipo } \\
\text { directivo, coordinadores, profesores, AMPA }\end{array}$ & $\begin{array}{l}\text { - Potencial formativo de las TIC } \\
\text { - Uso de las tabletas y móviles }\end{array}$ \\
\hline
\end{tabular}

Tabla 2. Dimensiones y sub-dimensiones de análisis de datos del centro

La Tabla 3 recoge las categorías que se utilizaron para la recogida y el análisis de las observaciones de aula, que se corresponden con la sub-dimensión Tipo de uso de las TIC en las aulas, de la dimensión Prácticas del centro con TIC de la Tabla 2.

\begin{tabular}{|l|l|}
\hline Dimensiones & Sub-dimensiones \\
\hline Datos de identificación & - Centro, curso/nivel, no de estudiantes, área/asignatura \\
\hline $\begin{array}{l}\text { Identificación de las macro- } \\
\text { actividades o tareas }\end{array}$ & - Tiempo dedicado a cada macro-actividad o tarea \\
\hline \multirow{2}{*}{ Actividades del alumnado con } & - Reproductiva o micro-tareas: realiza ejercicios o tareas \\
TIC & - Instrumental: aprende a manejar software \\
& $\begin{array}{l}\text { - Eúsqueda: búsqueda de información en Internet } \\
\text { Claboración: creación de productos escritos o visuales, }\end{array}$ \\
& - Proyectos propios que desarrolla el centro con TIC \\
\hline \multirow{2}{*}{ Rol del/ de la profesor/a } & - Exponer contenidos \\
& - Supervisar la realización de ejercicios o tareas \\
& - Proponer una actividad \\
\hline
\end{tabular}




\begin{tabular}{|l|l|}
\hline & - Orientar al alumnado en su aprendizaje \\
\hline \multirow{2}{*}{ Recursos utilizados } & - On line / Off line \\
& - Hardware y software \\
\hline \multirow{3}{*}{ Quien/es los utiliza/n } & - Profesor/a \\
& - Alumnado \\
& - Ambos \\
\hline \multirow{3}{*}{ Organización del alumnado } & - Individual \\
& - Pequeño grupo \\
& - Grupo clase \\
\hline
\end{tabular}

Tabla 3. Categorías para el análisis de las observaciones de aula.

A partir del informe de cada caso se elaboró la matriz individual de cada centro en cuanto a su grado de integración de las TIC en los ámbitos y dimensiones de la Tabla 4, lo cual permitió ubicar a cada centro en un estadio o fase particular. En los casos que un mismo centro se encontraba "a caballo" entre dos fases, es decir, en parte de las dimensiones se ubicaba en una fase y en parte de las dimensiones se ubicaba en otra adyacente, se diagnosticó entre las dos fases. De ahí que en la Tabla 5 aparezcan centros ubicados entre las fases de Iniciación-Aplicación y entre las fases de Aplicación-Integración.

\section{f. Una matriz de estadios o fases de un centro educativo en la transformación digital e integración pedagógica y organizativa de las TIC}

La matriz de análisis utilizada para diagnosticar el estadio en el que se encontraba cada centro escolar con relación al proceso de integración pedagógica y organizativa de las TIC fue elaborada por el equipo de EDULLAB de la Universidad de La Laguna a partir de diversos trabajos (Departamento de Educación, Universidades e Investigación del Gobierno Vasco, 2008; Severin 2011; Severin, Peirano y Falck, 2012; Lugo y Kelly, 2011; Padilha y Aguirre, 2011; Valverde, 2015). Dicha matriz se elaboró antes de realizar el análisis de datos de cada centro.

La matriz está constituida por cuatro niveles de transformación digital (Iniciación, Aplicación, Integración, y Transformación) (Figura 1) y por ocho dimensiones organizativas y pedagógicas del uso de las TIC en los centros (ver Tabla 4). De este modo, hemos podido describir e identificar la situación específica de cada centro en dimensiones concretas de práctica escolar con las TIC. Esta matriz no sólo ofrece un diagnóstico general del estadio en que se encuentra un centro con relación a las tecnologías, sino que también señala el estado de uso de las TIC en dimensiones concretas.

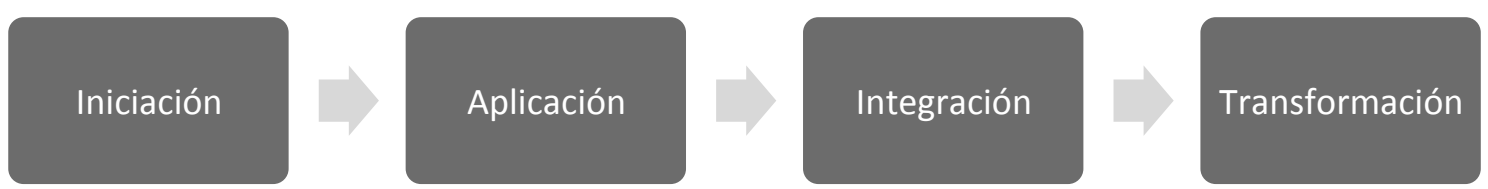

Figura 1. Etapas o estadios de desarrollo en la transformación digital de un centro escolar.

Fase de iniciación. A esta primera etapa o estadio de la transformación digital la hemos denominado fase de iniciación (Severin la llama de emergencia). En el ámbito pedagógico se caracteriza porque el centro no dispone de Plan TIC, ni desarrolla proyectos propios con TIC, ni participa en proyectos en red con otros centros. En cuanto al uso de las TIC en las aulas predominan clases centradas en el docente y los estudiantes tienen un acceso restringido a las tecnologías. El profesorado del centro no utiliza (o apenas muy poco) los recursos digitales. En el 
ámbito organizativo el centro no es visible en Internet (no dispone de web) o bien no es funcional ni operativa (no está actualizada). El colegio o instituto no se comunica con las familias por medio de las TIC. El profesorado no utiliza las TIC para comunicarse, ni para coordinarse.

Fase de Aplicación. Esta etapa supone en el ámbito pedagógico que el colegio o instituto tiene Plan TIC, pero no desarrolla proyectos propios con tecnologías, aunque algunos docentes participan en proyectos educativos en redes. Las clases están centradas en los docentes, las TIC se incorporan esporádicamente y los estudiantes tienen un acceso regular a las TIC. Los docentes utilizan materiales digitales de editoriales de libros de texto y repositorios institucionales, pero no elaboran materiales propios. En cuanto al ámbito organizativo el centro tiene una web o blog relativamente actualizado, participa en plataformas y redes institucionales y no institucionales, comienza a comunicarse con las familias por medio de las TIC, su profesorado también empieza a utilizarlas para comunicarse y coordinarse entre sí, y la mayoría los procesos administrativos se realizan con $\mathrm{TI}$, aunque no todo el profesorado sabe utilizarlos.

Fase de Integración. En el terreno pedagógico, esta etapa se caracteriza porque el centro tiene Plan TIC, desarrolla proyectos con las tecnologías y éstas se utilizan en las diversas áreas y asignaturas como recurso didáctico y para el desarrollo de la competencia digital, el centro participa en proyectos y redes educativas on line, las clases están centradas en los estudiantes, el docente asume un papel de animador del trabajo colaborativo de los estudiantes, los docentes elaboran y utilizan materiales digitales propios. En el ámbito organizativo el centro tiene web actualizada, y participa en plataformas y redes institucionales y no institucionales, el alumnado y las familias pueden acceder on line a determinada información o a blogs de grupo/clase y/o de asignaturas, el profesorado utiliza las TIC para la comunicación y el intercambio de información, y casi todos los procesos administrativos se realizan con TIC y casi todo el profesorado sabe utilizarlos.

Fase de transformación. Es la etapa más avanzada de maduración. Se caracteriza porque, en el aula y en el centro, los agentes participantes desarrollan prácticas con TIC con potencial pedagógico y social transformador, prácticas en las que se generan aprendizajes valiosos, conocimientos compartidos, y se desarrolla la autonomía y el pensamiento crítico. En el ámbito pedagógico el centro tiene un Plan TIC, que muestra una visión compartida del uso de las tecnologías en la escuela y en el que se contempla el desarrollo de diversos proyectos con tecnologías en los que está implicada toda la comunidad educativa, está implicado en proyectos y redes educativas online, constituye un entorno de aprendizaje permanente en el que alumnado y profesorado colaboran en la creación y comunicación de conocimiento, los docentes elaboran y utilizan recursos digitales, y los comparten en la red. En el ámbito organizativo el centro tiene web o blog actualizado, y participa en plataformas y redes sociales institucionales y no institucionales, está abierto permanentemente a través de Internet, dinamizando la comunicación educativa y una red social que abarca no solo a las familias sino a la sociedad en general, el profesorado utiliza las TIC como medio de comunicación y coordinación docente, y el centro hace un uso intensivo de las TIC en las tareas de gestión y administración en el que participa todo el profesorado.

\begin{tabular}{|l|l|}
\hline Ámbitos & Dimensiones \\
\hline \multirow{4}{*}{ PEDAGÓGICO } & Proyectos propios que desarrolla el centro con TIC \\
\cline { 2 - 2 } & $\begin{array}{l}\text { Participación del centro en otros proyectos, experiencias o redes } \\
\text { educativas on line }\end{array}$ \\
\cline { 2 - 2 } & $\begin{array}{l}\text { Modalidad de uso de las TIC en los procesos de enseñanza- } \\
\text { aprendizaje presencial y/o virtual }\end{array}$ \\
\cline { 2 - 2 } & $\begin{array}{l}\text { Producción y gestión de recursos digitales para la enseñanza y el } \\
\text { aprendizaje }\end{array}$ \\
\hline
\end{tabular}




\begin{tabular}{|l|l|}
\hline \multirow{5}{*}{ ORGANIZATIVO } & Visibilidad del centro en Internet \\
\cline { 2 - 2 } & $\begin{array}{l}\text { Comunicación con las familias y participación del AMPA en la política } \\
\text { TIC del centro }\end{array}$ \\
\cline { 2 - 2 } & $\begin{array}{l}\text { Utilización de las TIC para la comunicación y la coordinación docente } \\
\text { entre el profesorado del centro }\end{array}$ \\
\cline { 2 - 2 } & Utilización de las TIC para tareas administrativa y de gestión \\
\hline
\end{tabular}

Tabla 4. Ámbitos y dimensiones del uso de las TIC en los centros escolares

La matriz construida para el análisis tiene una doble entrada (Area, 2015). En el eje horizontal se encuentran los cuatro niveles de uso de las TIC en las prácticas educativas y en el eje vertical los ámbitos y dimensiones de uso de las tecnologías. En los dos primeros niveles van cambiando las tecnologías que se utilizan en las prácticas educativas, pero no hay cambios ni mejoras en las metodologías didácticas; mientras que en los dos últimos niveles van cambiando tanto las prácticas educativas como las tecnologías que se usan de tal manera que se transforma la forma de enseñar y de aprender. ¿Por qué en la última fase se habla de transformación? Porque las escuelas que persiguen un cambio más holístico e integral, y revisan sustancialmente el entorno de aprendizaje, han hecho uso de las tecnologías para transformarse radicalmente. En esta transformación todos los elementos del entorno de aprendizaje son nuevos y llevan a una nueva visión del aprendizaje y de la enseñanza (Groff, 2013).

Para la identificación de factores que facilitan e inhiben la integración de las TIC en los centros escolares comparamos los factores presentes en los centros ubicados en los extremos en cuanto a su nivel de integración. Esto es, en el caso de los CEIP comparamos los que se encontraban en la fase de iniciación y los que se ubicaban entre las fases de aplicación e integración; y en el caso de los IES comparamos los que se hallaban entre la fase de iniciación y aplicación, y los que se ubicaban en la fase de integración (Tabla 5).

\section{Resultados}

A continuación, presentamos los principales resultados en lo relativo al estadio de incorporación de las TIC en los centros estudiados identificando los factores y variables que inhiben la integración pedagógica y organizativa de las TIC en los centros escolares.

\section{a. Diagnóstico sobre el estadio o etapa de transformación digital en centros escolares públicos de Canarias}

1. Los centros estudiados no se encuentran en el mismo estadio de integración de las TIC en todas las dimensiones consideradas en el análisis, sino que se ubican en distintas fases, mayormente en fases adyacentes. Esto ha hecho que el proceso de análisis haya sido complejo y hayamos decidido ubicar a algunos centros no en un estadio sino entre dos (entre iniciación y aplicación; o entre aplicación e integración).

2. Según puede comprobarse la Tabla 5, los CEIP estudiados se concentran en los estadios intermedios, entre iniciación y aplicación, y entre aplicación e integración, mientras que los IES se concentran en el estadio intermedio entre iniciación y aplicación, y en el estadio de aplicación. Por otra parte, hay algunos CEIP en el estadio de iniciación y ninguno en el de integración, mientras que hay un solo IES en el estadio de iniciación y dos en el de integración. A partir de estos datos se 
puede concluir que la distribución de los CEIP y los IES de la muestra en los estadios de la matriz de integración de las TIC no es similar.

\begin{tabular}{|l|c|c|c|}
\hline \multirow{2}{*}{$\begin{array}{l}\text { Estadio de } \\
\text { transformación digital }\end{array}$} & \multicolumn{2}{|c|}{ Tipo de centro educativo } & \multirow{2}{*}{ Totales } \\
\cline { 2 - 3 } & CEIP/CEO & IES & \\
\hline INICIACIÓN & 5 & 1 & 6 \\
\hline Iniciación-Aplicación & 8 & 6 & 14 \\
\hline APLICACIÓN & 3 & 6 & 9 \\
\hline Aplicación-Integración & 7 & 2 & 9 \\
\hline INTEGRACIÓN & 0 & 2 & 2 \\
\hline TRANSFORMACIÓN & 0 & 0 & 0 \\
\hline
\end{tabular}

Tabla 5. Diagnóstico del estadio de integración de las TIC de los centros estudiados.

\section{b. Factores facilitadores e inhibidores de la transformación digital en centros escolares}

La realidad encontrada muestra una variedad de situaciones entre unos centros y otros debido, entre otras causas, a que cada centro es un microcosmos humano con su propia historia, experiencia y cultura particular. Existe un buen número de centros que inician el proceso de trabajo con las TIC, otros por el contrario se encuentren en situaciones de integración, más o menos avanzada, de integración de estas tecnologías en su funcionamiento. En el medio, la gran mayoría.

¿Por qué sucede esta diversidad de situaciones? En función de los datos recogidos y los análisis realizados respecto al estadio de integración de las TIC de cada centro, en este apartado intentaremos inferir cuáles son los factores que explican la situación particular en la que se encuentran los centros ubicados en cada extremo del continuo iniciación-integración. Con ello buscamos comprender los porqués de lo que ocurre, los motivos que explican que un centro escolar esté en un estadio inicial de aproximación al uso educativo de las TIC y otro sea usuario pleno e innovador con las mismas. Lo que pretendemos es dar respuesta a lo siguiente: ¿qué fenómenos o prácticas influyen en que un centro educativo integre, en distintos grados, las TIC a nivel de centro y de aula? Dicho de otro modo ¿cuáles son los factores que impulsan o facilitan el proceso de integración TIC, y cuáles lo dificultan o inhiben?

Nos planteamos la pregunta en ambos sentidos desde el supuesto de que, de esa manera, la respuesta permitirá identificar un rango más amplio de factores que si solo la abordamos desde una perspectiva o enfoque negativo. La Tabla 6 recoge la lista de factores facilitadores e inhibidores de la integración de las TIC en los centros escolares en relación con las dimensiones y sub-dimensiones de análisis (ver Tabla 2).

\begin{tabular}{|c|c|c|c|}
\hline Dimensiones & Sub-dimensiones & Factores facilitadores & Factores inhibidores \\
\hline $\begin{array}{l}\text { Características } \\
\text { generales del } \\
\text { centro }\end{array}$ & $\begin{array}{l}\text { - Características } \\
\text { descriptivas del centro } \\
\text { - Trayectoria innovadora } \\
\text { del centro } \\
\text { - Trayectoria del centro } \\
\text { respecto a las TIC }\end{array}$ & $\begin{array}{l}\text { - Cierta estabilidad del } \\
\text { claustro } \\
\text { - El carácter innovador } \\
\text { del centro, su cultura } \\
\text { innovadora } \\
\text { - Cierta trayectoria del } \\
\text { centro en el uso de las } \\
\text { TIC }\end{array}$ & $\begin{array}{l}\text { - La inestabilidad del claustro } \\
\text { - Una cultura organizativa que } \\
\text { tiene a la conservación de las } \\
\text { prácticas educativas y no a la } \\
\text { innovación } \\
\text { - La falta de experiencia del } \\
\text { centro en el uso de las TIC }\end{array}$ \\
\hline
\end{tabular}




\begin{tabular}{|c|c|c|c|}
\hline $\begin{array}{l}\text { Organización } \\
\text { y gestión de } \\
\text { los recursos } \\
\text { tecnológicos } \\
\text { del centro }\end{array}$ & $\begin{array}{l}\text { - Disponibilidad, } \\
\text { adquisición y } \\
\text { mantenimiento } \\
\text { - Organización y } \\
\text { distribución }\end{array}$ & $\begin{array}{l}\text { - La disponibilidad de } \\
\text { un conjunto de } \\
\text { recursos TIC, en } \\
\text { cantidad y calidad } \\
\text { suficiente, para que } \\
\text { el alumnado y el } \\
\text { profesorado tengan } \\
\text { acceso regular a los } \\
\text { mismos } \\
\text { - Una buena } \\
\text { conectividad } \\
\text { - - Una organización } \\
\text { de las TIC en el } \\
\text { centro que hace } \\
\text { posible que el } \\
\text { alumnado y el } \\
\text { profesorado tengan } \\
\text { un acceso regular a } \\
\text { las mismas }\end{array}$ & $\begin{array}{l}\text { - La escasez de dispositivos en } \\
\text { cantidad y calidad suficiente } \\
\text { para que el alumnado y el } \\
\text { profesorado tengan acceso } \\
\text { regular a los mismos } \\
\text { - Una conectividad limitada } \\
\text { - - Una organización de las TIC } \\
\text { en el centro que impide que } \\
\text { el alumnado y el profesorado } \\
\text { tengan un acceso regular a } \\
\text { las mismas }\end{array}$ \\
\hline $\begin{array}{l}\text { Política } \\
\text { interna del } \\
\text { centro } \\
\text { respecto a las } \\
\text { TIC }\end{array}$ & $\begin{array}{l}\text { - Características, } \\
\text { proceso y grado de } \\
\text { elaboración del Plan } \\
\text { TIC } \\
\text { - Liderazgo del Equipo } \\
\text { Directico en relación al } \\
\text { impulso de las TIC } \\
\text { - Coordinación, apoyo y } \\
\text { asesoramiento a la } \\
\text { integración de las TIC } \\
\text { - La visibilidad del centro } \\
\text { en internet } \\
\text { - La comunicación y } \\
\text { participación del AMPA en } \\
\text { la política TIC del centro }\end{array}$ & $\begin{array}{l}\text { - El apoyo del equipo } \\
\text { directivo a las } \\
\text { iniciativas que } \\
\text { desarrolla el } \\
\text { profesorado con TIC, } \\
\text { al trabajo del/de la } \\
\text { coordinador/a TIC y } \\
\text { el impulso de } \\
\text { proyecto } \\
\text { institucionales con } \\
\text { TIC de centro } \\
\text { - La presencia de, al } \\
\text { menos, un docente } \\
\text { convencido del valor } \\
\text { pedagógico de las } \\
\text { TIC que impulse su } \\
\text { uso en el centro por } \\
\text { medio de un conjunto } \\
\text { definido de acciones, } \\
\text { y realice su labor en } \\
\text { condiciones de } \\
\text { trabajo adecuadas } \\
\text { (ver Nota al pie de la } \\
\text { Tabla) } \\
\text { - El perfil y desarrollo } \\
\text { profesional de los } \\
\text { agentes que } \\
\text { desempeñan } \\
\text { funciones de } \\
\text { coordinación TIC está } \\
\text { orientado a lo } \\
\text { pedagógico más que }\end{array}$ & $\begin{array}{l}\text { - La falta de una visión } \\
\text { compartida del equipo } \\
\text { directivo acerca del potencial } \\
\text { educativo de las TIC } \\
\text { - La ausencia de algún docente } \\
\text { convencido del valor } \\
\text { pedagógico de las TIC que } \\
\text { impulse su uso en el centro } \\
\text { por medio de un conjunto } \\
\text { definido de acciones } \\
\text { - El perfil y desarrollo } \\
\text { profesional de los agentes } \\
\text { que desempeñan funciones } \\
\text { de coordinación TIC está } \\
\text { orientado a lo tecnológico } \\
\text { - La carencia de un liderazgo } \\
\text { distribuido que apoye y } \\
\text { dinamice la integración } \\
\text { pedagógica de las TIC } \\
\text { - El centro no dispone de Plan } \\
\text { de formación del centro o } \\
\text { dicho plan carece de acciones } \\
\text { que contribuyan a la } \\
\text { formación del profesorado en } \\
\text { la competencia digital } \\
\text { docente }\end{array}$ \\
\hline
\end{tabular}




\begin{tabular}{|c|c|c|c|}
\hline & & $\begin{array}{l}\text { a lo tecnológico } \\
\text { - Un liderazgo } \\
\text { distribuido, que tiene } \\
\text { ideas claras respecto } \\
\text { a la integración } \\
\text { pedagógica de las } \\
\text { TIC y toma } \\
\text { decisiones para } \\
\text { llevarlas a la práctica } \\
\text { en colaboración con } \\
\text { el profesorado } \\
\text { - La dinamización de la } \\
\text { formación del } \\
\text { profesorado en TIC } \\
\text { forma parte del Plan } \\
\text { de formación del } \\
\text { centro }\end{array}$ & \\
\hline $\begin{array}{l}\text { Prácticas del } \\
\text { centro con } \\
\text { TIC }\end{array}$ & $\begin{array}{l}\text { - Proyectos propios que } \\
\text { desarrolla el centro con } \\
\text { TIC } \\
\text { - Participación del centro } \\
\text { en otros proyectos, } \\
\text { experiencias o redes } \\
\text { educativas on line } \\
\text { - Tipo de uso de las TIC } \\
\text { en las aulas } \\
\text { - Producción y gestión } \\
\text { de recursos digitales } \\
\text { para la enseñanza y el } \\
\text { aprendizaje } \\
\text { - Uso de las TIC para } \\
\text { tareas administrativas, } \\
\text { de gestión y } \\
\text { comunicación entre el } \\
\text { profesorado }\end{array}$ & $\begin{array}{l}\text { - El centro participa } \\
\text { con otros centros y } \\
\text { organizaciones en } \\
\text { experiencias o } \\
\text { proyectos de uso } \\
\text { pedagógico de las } \\
\text { TIC (trabajo en red) } \\
\text { - Un profesorado } \\
\text { formado en el uso } \\
\text { pedagógico de las } \\
\text { TIC con metodologías } \\
\text { didácticas que ponen } \\
\text { el énfasis en el } \\
\text { aprendizaje } \\
\text { autónomo y el } \\
\text { trabajo colaborativo, } \\
\text { y con la capacidad de } \\
\text { adaptar recursos } \\
\text { digitales ajenos y } \\
\text { crear recursos } \\
\text { digitales propios }\end{array}$ & $\begin{array}{l}\text { - El centro no participa en } \\
\text { experiencias de uso } \\
\text { pedagógico de las TIC con } \\
\text { otros centros } \\
\text { - Un profesorado con carencias } \\
\text { y necesidades formativas en } \\
\text { el uso pedagógico de las TIC, } \\
\text { y en competencias para } \\
\text { adaptar recursos digitales } \\
\text { ajenos y crear recursos } \\
\text { digitales propios }\end{array}$ \\
\hline $\begin{array}{l}\text { Visiones de } \\
\text { los agentes } \\
\text { educativos }\end{array}$ & $\begin{array}{l}\text { - Potencial formativo de } \\
\text { las TIC } \\
\text { - Uso de las tabletas y } \\
\text { los móviles }\end{array}$ & & \\
\hline
\end{tabular}

Tabla 6. Factores facilitadores e inhibidores de la integración de las TIC de los centros estudiados. Nota. Por condiciones de trabajo adecuadas entendemos: tiempo de dedicación reconocido en el horario laboral; formación previa para el desarrollo de sus funciones, sobre todo de asesoramiento pedagógico al profesorado; y disponibilidad de asesoramiento tecnológico y pedagógico en su labor de coordinación. 


\section{Discusión y conclusiones}

Los resultados obtenidos en este estudio muestran que ningún centro de la muestra analizada se aproxima a una etapa de madurez, innovación permanente o de transformación usando como catalizador del cambio la tecnología y la cultura digital, y que solo dos centros educativos podemos identificarlos como centros que se encuentran en el nivel de integración. En concreto, los resultados revelan que la mayoría de los centros todavía no ha llegado a la fase de aplicación en todas las dimensiones (pedagógicas y organizativas) identificadas en la matriz de análisis (tabla 4).

Sin embargo, y en base a los resultados de otras investigaciones realizadas en Canarias (Area, 2010), puede afirmarse que la situación representa un avance relevante ya que, en todos los centros, las TIC son visibles, aunque en distintos grados, y ya han empezado a incidir en la vida organizativa y en las prácticas pedagógicas de sus docentes. No existe ningún centro ajeno o impermeable a la presencia tecnológica.

No obstante, y a pesar del progreso en la integración educativa de las TIC, los resultados evidencian la necesidad de seguir avanzado en la articulación de políticas educativas destinadas a facilitar la innovación pedagógica con las tecnologías digitales. En este sentido, parece indudable que, cualquier política educativa respecto a las TIC, que pretenda ser transformadora, deberá tener como principio facilitar que, a medio y largo plazo, todos los centros educativos públicos puedan ir caminando hacia estadios más avanzados de integración de la tecnología digital en su organización y práctica pedagógica.

Otro de los resultados destacados es la variabilidad de situaciones entre unos centros y otros con respecto a los niveles de integración de las TIC en las prácticas pedagógicas y organizativas. Esta variabilidad es debida, entre otras causas, a que cada centro tiene su propia historia, experiencia y cultura particular. Pero, indudablemente, para articular una política educativa TIC que permita avanzar en su integración educativa, se hace necesario identificar los factores que explican que un centro escolar esté en un estadio inicial de aproximación al uso educativo de las TIC y otro sea usuario pleno e innovador con las mismas. O dicho, en otros términos, es imprescindible identificar cuáles son los factores que impulsan o facilitan el proceso de transformación digital y educativa, y cuáles lo dificultan o inhiben.

¿Qué hemos aprendido? Este estudio nos ha permitido identificar una serie fenómenos de distinta naturaleza que se repiten en aquellos centros de un grado bajo o inicial de su transformación digital y educativa que consideramos inhiben, neutralizan o no facilitan dicho proceso. Estos fenómenos los hemos agrupado en torno a tres ámbitos o dimensiones de los centros escolares como organizaciones (González, 2003):

- Los factores y variables relacionados con la dimensión estructural del centro son:

- La escasez de dispositivos en cantidad y calidad suficiente para que el alumnado y el profesorado tengan acceso regular a los mismos

- La conectividad limitada que dificulta el trabajo en línea

- La inestabilidad del claustro

- Una organización de las TIC en el centro que impide que el alumnado y el profesorado tengan un acceso regular a las mismas

- Los agentes que desempeñan funciones de coordinación TIC tienen un perfil y desarrollo profesional orientado fundamentalmente a tareas de naturaleza tecnológica, disponen de escaso tiempo para el desempeño de sus funciones y carecen de formación adecuada para desarrollar funciones de asesoramiento en el uso pedagógico de las TIC 
Los factores y variables relativos a la dimensión relacional del centro son:

- La carencia de un liderazgo distribuido, que tenga ideas claras respecto a la integración pedagógica de las TIC y tome decisiones para llevarlas a la práctica en colaboración con el profesorado

- La ausencia de algún docente convencido del valor pedagógico de las TIC que impulse su uso en el centro por medio de un conjunto definido de acciones

Los factores negativos relacionados con las dimensiones procesual y cultural del centro son:

- La falta de una visión compartida del equipo directivo acerca del potencial educativo de las TIC

- La falta de experiencia del centro en el uso de las TIC

- La ausencia de proyectos o experiencias de uso pedagógico de las TIC con otros centros

- La ausencia de un plan de formación del profesorado del centro o que dicho plan carezca de acciones que contribuyan a la formación del profesorado en la competencia digital docente

- El profesorado presenta carencias y necesidades formativas en el uso pedagógico de las TIC, y en competencias para adaptar recursos digitales ajenos y crear recursos digitales propios

- Una cultura organizativa que tiende a la conservación de las prácticas educativas y no a la innovación

¿Cuáles son las implicaciones de este estudio para la política educativa y la práctica escolar? En primer lugar, entendemos que, en el caso de la escuela pública, que es objeto de este estudio, las Administraciones Educativas -tanto la estatal como la autonómica- son los primeros agentes responsables de evitar, en lo posible, que estos factores ejerzan su influjo inhibidor. En el caso de la adquisición y mantenimiento de equipos, y en la mejora de la conectividad, buena parte de los centros estudiados -sobre todo en el primer asunto- están buscando y encontrando soluciones e invirtiendo de entre los pocos recursos con que cuentan. No obstante, quisiéramos reiterar que, si se demanda una mayor integración de las TIC por parte de los centros y el profesorado, esta debe ir precedida y acompañada de una mayor implicación de las autoridades públicas en contrarrestar los factores estructurales y facilitar las condiciones para el desarrollo óptimo en los centros de los factores relacionales, procesuales y culturales. En este sentido, los resultados de este estudio fueron útiles para la planificación de nuevas medidas de la política educativa desarrollada en Canarias en el marco del referido programa TSP (Tecnologías al Servicio de las Personas) especialmente con relación a la figura del profesor coordinador de centro escolar.

En segundo lugar, queremos referirnos al potencial de la matriz de análisis creada como instrumento de autodiagnóstico que puede ser utilizada por la comunidad docente de un centro con la finalidad de identificar el estadio o fase en la que se sitúa, y detectar cuál o cuáles son las dimensiones pedagógicas y organizativas en las que se encuentra más deficitario. Este autodiagnóstico permitirá elaborar aquellos planes de mejora y actuar directamente sobre dichas dimensiones cara a seguir evolucionando en el proceso de madurez organizativa del centro con relación a su transformación digital.

Finalmente, sugerimos que la continuidad de este estudio tiene dos líneas de trabajo complementarias. Por una parte, sería necesario extender este análisis a otros centros y contextos geográficos diferentes para confirmar si se identifican los mismos factores que potencian o inhiben 
la innovación pedagógica con TIC. Por otra, sería interesante volver a repetir el estudio sobre una muestra de los centros que ya participaron en el mismo con la finalidad de analizar si se han producido cambios y avances en su madurez o transformación digital como organización educativa e indagar los posibles motivos que los han propiciado.

\section{Reconocimientos}

Esta investigación fue financiada en el marco del Plan de Evaluación del Proyecto TSP (Tecnologías al Servicio de las Personas) por la Consejería de Educación del Gobierno de Canarias y que obtuvo, en concurso público, la Fundación General de la Universidad de La Laguna encargándose de su ejecución el grupo Laboratorio de Educación y Nuevas Tecnologías (EDULLAB) de la Universidad de La Laguna.

\section{Referencias}

Area, M. (2010). El proceso de integración y uso pedagógico de las TIC en los centros educativos. Un estudio de casos. Revista de educación, 352, 77-97.

Area, M. (Dir.) (2015). La integración educativa de las TIC en el sistema escolar de Canarias: las visiones y prácticas de los agentes educativos. Informe final de evaluación TSP. Repositorio institucional de la Universidad de La Laguna. URI: http://riull.ull.es/xmlui/handle/915/13405

Balanskat, A., Blamire, R., y Kefala, S. (2006). The ICT impact report: A review of studies of ICT impact on schools in Europe. Brussels: European Communities.

British Educational Communications and Technology Agency (2004). A review of the research literature on barriers to the uptake of ICT by teachers. BECTA. URI: http://dera.ioe.ac.uk/id/eprint/1603

Departamento de Educación, Universidades e Investigación (2008). Modelo de Madurez Tecnológica del Centro Educativo. Dirección de Innovación Pedagógica. Departamento de Educación, Universidades e Investigación del Gobierno Vasco.

Ertmer, P. (1999). Addressing first- and second-order barriers to change: Strategies for technology integration. Educational Technology Research and Development, 47(4), 47-61. https://doi.org/10.1007/BF02299597

Gale, M., y Aarons, C. (2018). Digital Transformation. Delivering on the Promise. Leader to Leader, 90, 30-36.

González, Ma T. (Coord.) (2003). Organización y Gestión de Centros Escolares. Dimensiones y Procesos. Madrid: Pearson Educación.

Groff, J. (2013). Technology-Rich Innovative Learning Environments. Paris: OECD-CERI. Working Paper. Recuperado de: http://www.oecd.org/education/ceri/TechnologyRich\%20Innovative\%20Learning\%20Environments\%20by\%20Jennifer\%20Groff.pdf

Hew, K.F., y Brush, T. (2007). Integrating technology into K-12 teaching and learning: current knowledge gaps and recommendations for future research. Educational Technology Research and Development, 55, 223-252. https://doi.org/10.1007/s11423-006-9022-5

Kampylis, P. Punie, Y., y Devine, J. (2015). Promoting Effective Digital-Age Learning - A European Framework for Digitally-Competent Educational Organisations. European Union. DOI:10.2791/54070

Keengwe, J., Onchwari, G., y Wachira, P. (2008). Computer technology integration and student learning: barriers and promise. Journal of Science Education and Technology, 17 (2008), 560-565. https://doi.org/10.1007/s10956-008-9123-5

Kopcha, T. J. (2012). Teachers' perceptions of the barriers to technology integration and practices with technology under situated professional development. Computers \& Education, 59, 1109-1121. https://doi.org/10.1016/j.compedu.2012.05.014 
Law, N., Yuen, A., y Fox, R. (2011). Educational Innovations Beyond Technology. Nurturing Leadership and Establishing Learning Organizations. New York: Springer.

Leignel A., Ungaro, J.L., y Staar, T. (2016). Digital Transformation: Information System Governance (Vol 6). London: ISTE/Honboken, NJ: John Wiley \& Sons.

Lugo, Ma T., y Kelly, V. (Coords.) (2011). La matriz TIC. Una herramienta para planificar las Tecnologías de la Información y Comunicación en las instituciones educativas. Buenos Aires: Instituto Internacional de Planeamiento de la Educación-IIPE-UNESCO. Recuperado de: http://www.webinar.org.ar/sites/default/files/actividad/documentos/Articulo\%20mat riz\%20TIC.pdf

Matt, C., Hess, T., y Benlian, A. (2015). Digital Transformation Strategies. Business and Information Systems Engineering, 57(5), 339-343. DOI 10.1007/s12599-015-0401-5

Padilha, M., y Aguirre, S. (2011). La integración de las TIC en la escuela. Indicadores cualitativos y metodología de investigación. Organización Estados Iberoamericanos-IDIE TIC Brasil/Fundación Telefónica. Recuperado de: http://www.oei.es/idie/IntegracionTIC.pdf

Pelgrum, W.J. (2001). Obstacles to the integration of ICT in education: Results from a worldwide educational assessment. Computers and Education, 37, 163-178. https://doi.org/10.1016/S0360-1315(01)00045$\underline{8}$

Severin, E. (2011). Tecnologías para la Educación. Un Marco para la Acción. Notas Técnicas \# IDB-TN-358. Banco Interamericano de Desarrollo. División de Educación. Recuperado de: http://idbdocs.iadb.org/wsdocs/getdocument.aspx?docnum =36613530.

Severin, E., Peirano, C., y Falck, D. (2012). Guía Básica para la Evaluación de Proyectos. Notas Técnicas \# IDBTN-358. Banco Interamericano de Desarrollo. División de Educación. Recuperado de: http://idbdocs.iadb.org/wsdocs/getdocument. aspx?docnum $=36665384$.

Valverde, J. (Coord.) (2015). El proyecto de educación digital en un centro educativo. Madrid, Síntesis. 